\title{
VALORES DE REFERENCIA DE TEST MOTORES EN ESQUIADORES ALPINOS ESPAÑOLES DE 11 A 14 AÑOS
}

\author{
MOTOR TEST REFERENCE VALUES IN SPANISH ALPINE SKIERS FROM 11 \\ TO 14 YEARS OLD
}

\begin{abstract}
Pablo Ruiz de Almiron-Megías 1; Pablo J. Gómez-López ${ }^{2}$; Daniel Mayorga-Vega ${ }^{3}$
${ }^{1}$ Facultad de Ciencias del Deporte, Universidad Europea de Madrid, Madrid (España)

${ }^{2}$ Departamento de Educación Física y Deportiva, Facultad de Ciencias del Deporte, Universidad de Granada, Granada (España)

${ }^{3}$ Departamento de Didáctica de la Expresión Musical, Plástica y Corporal, Facultad de Humanidades y Ciencias de la Educación, Universidad de Jaén, Jaén (España)
\end{abstract}

Detalles del artículo:

Número de palabras: 6948; Tablas: 3; Figuras: 11; Referencias: 31

Recibido: marzo 2019; Aceptado: mayo 2019; Publicado: julio 2019

Conflicto de interés: El autor declara que no existen conflictos de interés.

Correspondencia del autor: Pablo Ruiz de Almiron-Megías, pablo@mamutsierranevada.com

\section{Resumen}

Introducción: Los objetivos del estudio fueron mostrar los valores percentiles de diferentes test motores en esquiadores españoles de categoría infantil y compararlos con sus niveles de rendimiento entre los diferentes grupos de edad y sexo. Metodología: 135 esquiadores españoles (53 niñas y 82 niños) de 11-14 años de edad y categoría infantil, realizaron 11 test motores: 1) Flexión de tronco adelante; 2) Flamenco; 3) Hexágono; 4) Circuito obstáculos de Harre; 5) 5 x 10 m; 6) Salto de longitud; 7) Squat Jump; 8) Counter Movement Jump; 9) Drop Jump; 10) Saltos verticales continuos durante 15 segundos, y 11) 45 segundos. Resultados: Los resultados del ANOVA de dos factores mostraron numerosas diferencias estadísticamente significativas entre las categorías de edad y sexo. Conclusiones: La elección de las pruebas utilizadas para la predicción del rendimiento deportivo en edad infantil en este estudio puede ser útiles para evaluar las capacidades físicas y controlar el entrenamiento en el esquí alpino en la edad de 11-14 años.

Palabras claves: Evaluación condición física, esquí alpino, entrenamiento con niños, edad escolar 


\begin{abstract}
Introduction: The objectives of the study were to show the percentile values of different motor tests in Spanish children skiers and compare them with their performance levels between the different age and sex groups. Methodology: 135 Spanish skiers (53 girls and 82 boys) of 11-14 years of age and children, performed 11 motor tests: 1) Flexion of the trunk forward; 2) Flamenco; 3) Hexagon; 4) Harre obstacles circuit; 5) 5 x 10 m; 6) Long jump; 7) Squat Jump; 8) Counter Movement Jump; 9) Drop Jump; 10) Continuous vertical jumps for 15 seconds, and 11) 45 seconds. Results: The two-factor ANOVA results showed numerous statistically significant differences between the age and sex categories. Conclusions: The choice of tests used for the prediction of athletic performance in children in this study can be useful to assess physical abilities and control training in alpine skiing at the age of 11-14 years.
\end{abstract}

Key words: Physical condition assessment, alpine skiing, training with children, school age

\title{
INTRODUCCIÓN
}

El rendimiento deportivo en esquí alpino depende del nivel de las habilidades motrices específicas adquiridas (técnica y táctica), de la capacidad motora general, de las capacidades funcionales de velocidad, fuerza, coordinación, equilibrio (Neumayr et al., 2003), así como resistencia a la velocidad (Bosco, 1997, 2000).

Existen numerosos intentos de evaluar los atributos físicos y fisiológicos en esquí alpino (Neumayr et al., 2003; Turnbull, Kilding \& Keogh, 2009; Bandalo \& Lesnik, 2011), si bien son en edad adulta y juvenil. Algunos países establecen valores de referencia a partir de las marcas obtenidas en la realización de diferentes baterías de test en esquiadores. Entre estos destacamos las baterías de test Alpine Medal Test realizada con esquiadores americanos (USSA, 2005; Stielow, 2010), Swiss Ski Power Test realizada con esquiadores suizos (Gorski, Rosser, Hoppeler \& Vogt, 2014), los valores de referencia con esquiadores austriacos (Müller, Hildebrandt \& Raschner, 2015; Müller, Müller, Kornexl \& Raschner, 2015; Steidl-Müller, Hildebrandt, Müller, Fink \& Raschner, 2017; Steidl-Müller, Hildebrandt, Raschner \& Müller, 2019) y las realizadas con esquiadores noruegos con la batería Ironman Test (NSF, 2014). En cambio, no se conocen baterías de test ni valores de referencia en deportistas de categoría infantil.

En España, no existe una batería de test ni valores de referencia suficientemente probados como los referidos en los casos anteriores. Solo encontramos algunos estudios de validación de pruebas físicas y fisiológicas relacionadas con el rendimiento (Ruiz de Almirón, Mesa-Mesa, Gómez-López, \& Gutiérrez, 2002; Gómez-López, Rupérez, Ruiz de AlmirónMegías, \& Calderon, 2012). Por este motivo, los objetivos del estudio fueron mostrar los valores percentiles de diferentes test motores en esquiadores españoles de categoría infantil y compararlos con sus niveles de rendimiento entre los diferentes grupos de edad y sexo.

\section{MATERIAL Y MÉTODOS}

\section{Diseño}

Se realizó un estudio transversal en esquiadores de diferente nivel de rendimiento a los que se evaluaron capacidades físicas relacionadas con el deporte mediante diferentes test 
motores para descubrir diferencias existentes por grupos de edad y sexo, que pueden servir de referencia en el esquí alpino de categoría infantil español.

\section{Participantes}

Un total de 135 esquiadores (82 niños y 53 niñas) de 11-14 años de edad participaron en el estudio. Todos eran esquiadores seleccionados por sus federaciones para representarles en los Campeonatos de España Infantil que se celebraron en Sierra Nevada en la temporada 20002001. Todos se encontraban en el ranking nacional RFEDI (Real Federación Española de Deportes de Invierno) de categoría infantil y realizaron entre 12-18 competiciones de ámbito autonómico, nacional e internacional durante esta temporada.

\section{Medidas}

Se aplicaron 11 test motores bajo las mismas condiciones (humedad, temperatura, altitud, hora del día y orden de ejecución), utilizándose el mismo material, en las mismas instalaciones y realizadas por el mismo personal experimentado. Todos los participantes realizaron las pruebas con calzado e indumentaria deportiva.

Los test por orden de aplicación fueron los siguientes: 1) Flexión de tronco adelante desde la posición de sentado (o test sit-and-reach clásico) para evaluar la flexibilidad (tangente de los pies en cm desde el borde exterior del cajón, con apoyo de los pies descalzos) (Council of Europe, 1993); 2) Flamenco para el equilibrio estático (número de caídas) (Council of Europe, 1993); 3) Hexágono para valorar la capacidad de equilibrio dinámico-coordinación (s) (Dillman, 1990); 4) Circuito de obstáculos de Harre para la coordinación dinámica general (s) (Müller y col., 2015a); 5) 5 x 10 m para evaluar de velocidad-coordinación en desplazamiento (s) (Council of Europe, 1993); 6) Salto de longitud para la fuerza explosiva (cm) (Gorski et al., 2014); 7) Salto vertical Squat Jump de Bosco (SJ) para la fuerza explosiva (Ergojump-Bosco System. Brevetto, Italia) (altura de salto en $\mathrm{cm}$ ) (Bosco, 1990); 8) Salto vertical Counter Movement Jump (CMJ) para la fuerza explosivo-elástica (altura de salto en cm) (Bosco, 1990); 9) Salto vertical Drop Jump de Bosco (DJ) para la capacidad de fuerza reactiva (altura de salto en $\mathrm{cm}$ ) (Bosco, 1990); 10) Saltos verticales continuos durante 15 segundos de Bosco (CMJ15) para la capacidad de resistencia a la velocidad durante 15 segundos saltando (altura de saltos en $\mathrm{cm}$ ) (Bosco, 1990), y 11) Saltos verticales continuos durante 45 segundos de Bosco (CMJ45) para la capacidad de resistencia a la velocidad durante 45 segundos saltando (altura de saltos en $\mathrm{cm}$ ) (Bosco, 1990). Todas las pruebas se aplicaron de acuerdo al protocolo original, el cual puede ser consultado en las referencias indicadas en cada prueba.

\section{Procedimiento}

El protocolo del presente estudio fue aprobado por el Comité de Ética de la Universidad de Granada. Los entrenadores, tutores legales y participantes dieron su consentimiento informado para realizar el estudio. Los datos se recogieron durante la celebración del Campeonato Nacional de Esquí Alpino en la temporada 2000-2001. Las sesiones de evaluación se planificaron y acordaron previamente con los responsables de las selecciones autonómicas. La evaluación se realizó en dos sesiones de tarde durante dos días diferentes previos al campeonato. En la primera sesión se llevaron a cabo los test 1-6, y en la segunda sesión los test 7-11. Los participantes debían asistir a las sesiones de evaluación con un descanso mínimo de 24 horas y no habiendo ingerido alimento al menos 3 horas antes del inicio de la sesión. 


\section{Análisis estadísticos}

Se calcularon estadísticos descriptivos de las variables medidas. Luego se calcularon los valores de los percentiles (de cinco en cinco) de cada una de las variables medidas separados por sexo. Finalmente, la comparación de los niveles de capacidad física entre los diferentes grupos de edad y sexo se estudió mediante un ANOVA de dos factores (edad y sexo). Posteriormente, para los análisis post hoc, se utilizó las comparaciones por pares con la corrección de Bonferroni. Todos los análisis estadísticos se realizaron con el programa SPSS, versión 21.0 para Windows (IBM ${ }^{\circledR}$ SPSS ${ }^{\circledR}$ Statistics). El nivel de significación se estableció en valores de $\mathrm{p}<0,05$.

\section{RESULTADOS}

La Tabla 1 muestra las características generales de los participantes en el estudio.

Tabla 1. Características generales de los esquiadores de esquí alpino de categoría infantil estudiados

\begin{tabular}{ccccccccc}
\hline \multicolumn{5}{c}{ Mujeres $(N=53)$} & \multicolumn{5}{c}{ Varones $(N=82)$} \\
\hline Grupo & $n$ & Edad (años) & Altura (cm) & Peso $(\mathrm{kg})$ & $n$ & Edad (años) & Altura (cm) & Peso (kg) \\
\hline 11 & 10 & $11,7(0,2)$ & $145,1(6,0)$ & $36,5(8,4)$ & 17 & $11,7(0,3)$ & $146,2(6,0)$ & $36,9(8,2)$ \\
12 & 17 & $12,4(0,6)$ & $148,6(6,0)$ & $37,8(8,2)$ & 24 & $12,6(0,3)$ & $147,8(6,0)$ & $38,5(6,2)$ \\
13 & 11 & $13,7(0,2)$ & $156,0(6,0)$ & $46,3(7,4)$ & 21 & $13,8(0,2)$ & $154,0(9,0)$ & $44,3(9,7)$ \\
14 & 15 & $14,3(0,5)$ & $161,9(6,0)$ & $50,4(8,2)$ & 20 & $14,5(0,4)$ & $159,2(12,0)$ & $48,1(9,3)$ \\
\hline
\end{tabular}

Nota. Los datos están reportados como media y desviación estándar (esta última entre paréntesis)

En las Tablas 2-3 se muestran los valores de los percentiles (de cinco en cinco) para cada test en esquiadores españoles de categoría infantil (11-14 años) en niñas y niños, respectivamente. Para el test de flamenco, hexágono, circuito obstáculos de Harre, y 5 x 10 m menores valores indican un mejor rendimiento. Por tanto, los percentiles tienen que interpretarse en el sentido opuesto (es decir, un percentil 5 es mejor que 10, etc.). 
Tabla 2. Percentiles para cada test en niñas esquiadoras españolas de 11 a 14 años.

\begin{tabular}{|c|c|c|c|c|c|c|c|c|c|c|}
\hline & $\begin{array}{l}\text { Flexión } \\
\text { de } \\
\text { tronco } \\
(\mathrm{cm})\end{array}$ & $\begin{array}{c}\text { Flamenco } \\
\qquad\left(\mathrm{n}^{\circ}\right. \\
\text { caídas })\end{array}$ & $\begin{array}{l}\text { Hexágono } \\
\text { (s) }\end{array}$ & $\begin{array}{l}\text { Harre } \\
(\mathrm{s})\end{array}$ & $\begin{array}{c}5 \times 10 \\
\mathrm{~m} \\
(\mathrm{~s})\end{array}$ & $\begin{array}{l}\text { Salto de } \\
\text { longitud } \\
\quad(\mathrm{cm})\end{array}$ & $\begin{array}{c}\mathrm{SJ} \\
(\mathrm{cm})\end{array}$ & $\begin{array}{l}\text { CMJ } \\
(\mathrm{cm})\end{array}$ & $\begin{array}{l}\text { Drop } \\
\text { Jump } \\
(\mathrm{cm})\end{array}$ & $\begin{array}{c}\text { CMJ15 } \\
(\mathrm{cm})\end{array}$ \\
\hline Percentiles & $n=38$ & $n=45$ & $n=53$ & $\begin{array}{l}n= \\
45\end{array}$ & $\begin{array}{c}n= \\
53\end{array}$ & $n=45$ & $\begin{array}{c}n= \\
13\end{array}$ & $\begin{array}{c}n= \\
16\end{array}$ & $n=8$ & $n=8$ \\
\hline 95 & 30,0 & 0,0 & 9,1 & 12,5 & 18,0 & 213,5 & 38,0 & 37,0 & 31,8 & 22,5 \\
\hline 90 & 29,1 & 0,0 & 9,3 & 12,9 & 18,5 & 207,0 & 36,8 & 34,2 & 31,8 & 22,5 \\
\hline 85 & 28,2 & 1,0 & 9,4 & 13,1 & 18,6 & 195,5 & 34,7 & 32,3 & 31,2 & 22,3 \\
\hline 80 & 27,1 & 1,0 & 10,3 & 13,2 & 18,7 & 194,0 & 32,6 & 31,5 & 30,5 & 22,2 \\
\hline 75 & 25,5 & 1,5 & 10,5 & 13,5 & 18,8 & 190,0 & 31,7 & 30,9 & 29,8 & 21,3 \\
\hline 70 & 22,0 & 3,0 & 10,8 & 13,7 & 19,1 & 190,0 & 31,3 & 30,6 & 29,1 & 19,8 \\
\hline 65 & 21,0 & 3,0 & 11,0 & 13,9 & 19,3 & 185,0 & 30,7 & 30,4 & 28,6 & 18,6 \\
\hline 60 & 21,0 & 3,4 & 11,2 & 14,0 & 19,6 & 180,0 & 30,5 & 29,6 & 28,4 & 17,9 \\
\hline 55 & 20,0 & 4,0 & 11,3 & 14,1 & 19,6 & 180,0 & 29,8 & 29,1 & 27,9 & 17,2 \\
\hline 50 & 19,0 & 4,0 & 11,4 & 14,3 & 19,9 & 175,0 & 28,1 & 28,9 & 26,1 & 17,1 \\
\hline 45 & 19,0 & 4,3 & 11,5 & 14,6 & 20,3 & 170,0 & 27,7 & 28,5 & 24,2 & 17,0 \\
\hline 40 & 19,0 & 5,0 & 11,6 & 14,9 & 20,5 & 170,0 & 27,0 & 27,9 & 23,7 & 15,8 \\
\hline 35 & 18,3 & 5,0 & 11,7 & 15,0 & 20,7 & 160,5 & 25,9 & 27,8 & 23,3 & 14,5 \\
\hline 30 & 16,7 & 5,0 & 12,1 & 15,2 & 20,8 & 160,0 & 25,5 & 26,5 & 22,2 & 13,9 \\
\hline 25 & 16,0 & 6,0 & 12,8 & 15,6 & 20,9 & 155,0 & 24,3 & 24,8 & 20,6 & 13,7 \\
\hline 20 & 13,8 & 6,0 & 12,9 & 15,9 & 21,1 & 147,0 & 23,0 & 23,7 & 19,7 & 13,3 \\
\hline 15 & 10,1 & 7,1 & 13,1 & 16,0 & 21,5 & 139,5 & 22,0 & 21,6 & 19,5 & 12,6 \\
\hline 10 & 2,9 & 8,0 & 13,6 & 16,3 & 22,0 & 130,0 & 19,9 & 19,1 & 19,4 & 12,0 \\
\hline 5 & 1,0 & 13,5 & 14,6 & 17,6 & 22,7 & 130,0 & 18,6 & 18,6 & 19,4 & 12,0 \\
\hline
\end{tabular}

Nota. SJ = Squat Jump; CMJ = Counter Movement Jump; CMJ15 = Saltos verticales continuos durante 15 segundos de Bosco. 
Tabla 3. Percentiles para cada test en niños esquiadores españoles de 11 a 14 años.

\begin{tabular}{|c|c|c|c|c|c|c|c|c|c|c|}
\hline & $\begin{array}{l}\text { Flexión } \\
\text { de } \\
\text { tronco } \\
(\mathrm{cm})\end{array}$ & $\begin{array}{c}\text { Flamenco } \\
\qquad\left(\mathrm{n}^{\circ}\right. \\
\text { caídas })\end{array}$ & $\begin{array}{l}\text { Hexágono } \\
\text { (s) }\end{array}$ & $\begin{array}{l}\text { Harre } \\
\text { (s) }\end{array}$ & $\begin{array}{c}5 \times 10 \\
\mathrm{~m} \\
(\mathrm{~s})\end{array}$ & $\begin{array}{l}\text { Salto de } \\
\text { longitud } \\
\quad(\mathrm{cm})\end{array}$ & $\begin{array}{c}\text { SJ } \\
(\mathrm{cm})\end{array}$ & $\begin{array}{l}\mathrm{CMJ} \\
(\mathrm{cm})\end{array}$ & $\begin{array}{c}\text { Drop } \\
\text { Jump } \\
(\mathrm{cm})\end{array}$ & $\begin{array}{c}\text { CMJ } \\
15 \\
(\mathrm{~cm})\end{array}$ \\
\hline $\begin{array}{l}\text { Percentile } \\
\mathrm{s}\end{array}$ & $n=59$ & $n=77$ & $n=82$ & $\begin{array}{c}n= \\
77\end{array}$ & $\begin{array}{c}n= \\
82\end{array}$ & $n=77$ & $\begin{array}{l}n= \\
23\end{array}$ & $\begin{array}{l}n= \\
25\end{array}$ & $\begin{array}{l}n= \\
20\end{array}$ & $\begin{array}{c}n= \\
20\end{array}$ \\
\hline 95 & 28,0 & 0,0 & 8,8 & 11,7 & 16,1 & 235,0 & 43,4 & 45,4 & 45,2 & 28,6 \\
\hline 90 & 26,0 & 0,0 & 9,1 & 12,0 & 17,5 & 221,0 & 40,0 & 42,7 & 42,9 & 25,1 \\
\hline 85 & 25,5 & 1,0 & 9,4 & 12,2 & 17,8 & 211,5 & 36,9 & 39,2 & 39,3 & 24,8 \\
\hline 80 & 24,0 & 1,0 & 9,9 & 12,4 & 18,0 & 205,0 & 36,5 & 38,5 & 38,8 & 23,7 \\
\hline 75 & 23,5 & 1,5 & 10,1 & 12,8 & 18,3 & 205,0 & 35,0 & 37,3 & 38,0 & 22,9 \\
\hline 70 & 22,0 & 2,0 & 10,4 & 12,9 & 18,5 & 200,0 & 34,0 & 36,2 & 37,2 & 22,6 \\
\hline 65 & 20,0 & 3,0 & 10,5 & 13,1 & 18,6 & 198,5 & 33,5 & 35,6 & 34,6 & 22,2 \\
\hline 60 & 19,0 & 3,0 & 10,6 & 13,5 & 18,8 & 194,0 & 32,4 & 34,1 & 33,3 & 21,9 \\
\hline 55 & 18,0 & 3,0 & 10,9 & 13,6 & 19,0 & 189,5 & 31,8 & 33,1 & 33,0 & 21,2 \\
\hline 50 & 17,0 & 4,0 & 11,1 & 13,7 & 19,3 & 185,0 & 31,7 & 32,3 & 32,5 & 20,4 \\
\hline 45 & 16,0 & 4,0 & 11,4 & 13,9 & 19,5 & 185,0 & 31,1 & 31,8 & 32,0 & 20,2 \\
\hline 40 & 16,0 & 5,0 & 11,6 & 14,2 & 19,5 & 180,0 & 30,6 & 30,9 & 31,2 & 19,8 \\
\hline 35 & 15,0 & 5,0 & 11,8 & 14,6 & 19,8 & 176,5 & 29,8 & 30,6 & 30,0 & 19,4 \\
\hline 30 & 14,0 & 6,0 & 12,1 & 15,0 & 20,1 & 175,0 & 28,9 & 30,1 & 29,2 & 19,2 \\
\hline 25 & 13,0 & 6,0 & 12,5 & 15,3 & 20,6 & 170,0 & 28,0 & 28,7 & 28,8 & 19,1 \\
\hline 20 & 11,0 & 7,0 & 12,9 & 15,5 & 20,7 & 165,0 & 25,8 & 28,3 & 28,2 & 19,0 \\
\hline 15 & 8,0 & 7,3 & 13,2 & 16,0 & 21,1 & 160,0 & 24,1 & 27,5 & 26,6 & 17,3 \\
\hline 10 & 5,0 & 8,0 & 13,9 & 16,5 & 21,6 & 155,0 & 22,3 & 25,3 & 23,4 & 16,3 \\
\hline 5 & 2,5 & 11,4 & 15,4 & 17,3 & 22,1 & 150,0 & 20,6 & 22,7 & 22,3 & 14,9 \\
\hline
\end{tabular}

Nota. $\mathrm{SJ}=$ Squat Jump; CMJ = Counter Movement Jump; CMJ15 = Saltos verticales continuos durante 15 segundos de Bosco. 
La Figura 1 muestra la comparación de los valores del test de flexión de tronco $(\mathrm{cm})$ entre los grupos de edad y sexo. Los resultados mostraron un efecto de interacción estadísticamente significativo $(p<0,05)$. Posteriormente, las comparaciones por pares mostraron que los esquiadores de 13 y 14 años tenían unos niveles estadísticamente significativos mayores que sus compañeros de 11 años $(p<0,01)$; las esquiadoras de 12 y 13 años tenían unos niveles estadísticamente significativos mayores que sus compañeras de 11 años $(p<0,001)$. En cuanto a la comparación por sexo, los resultados mostraron que las esquiadoras de 13 años tenían unos valores estadísticamente mayores que los esquiadores de su mismo grupo de edad $(p<0,05)$.

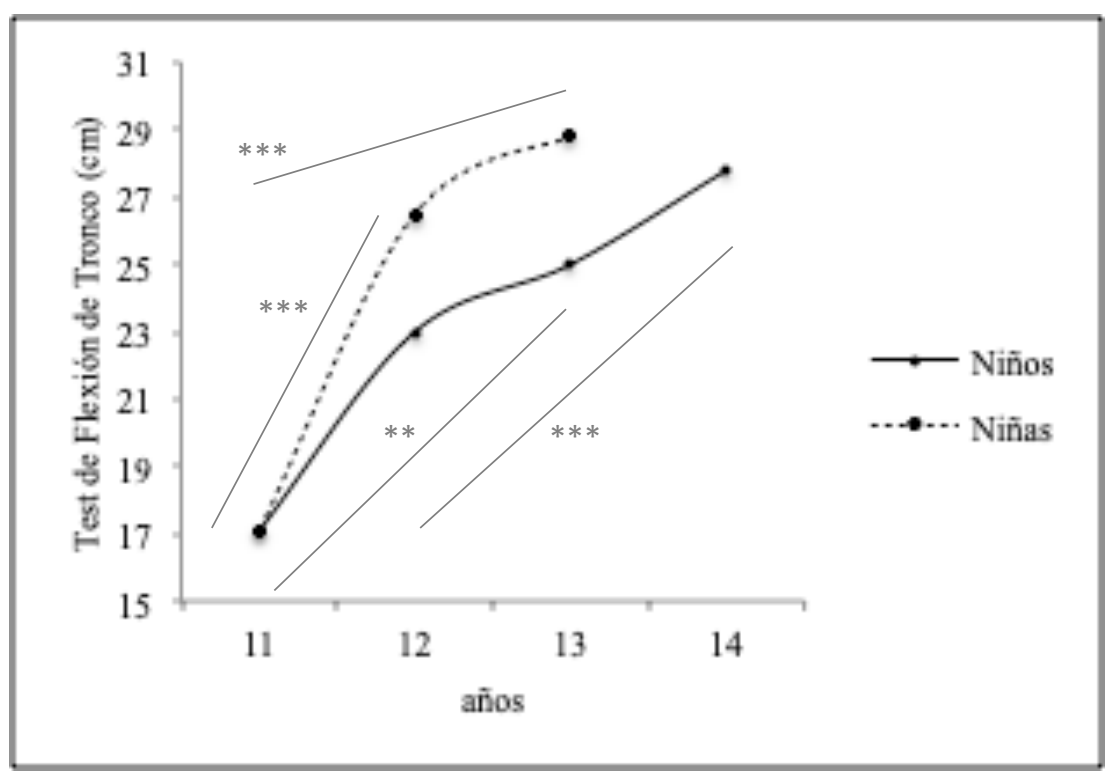

Figura 1. Comparación de los valores del test de flexión de tronco $(\mathrm{cm})$ entre los grupos de edad y sexo. Los valores representan la media y las barras de error el error estándar. Nivel de significación del análisis de la varianza de dos factores $(p<0,05)$ seguido por las comparaciones por pares con la corrección de Bonferroni (sobre figura): $* p<0,05, * * p<0,01 \mathrm{y} * * * p<0,001$.

La Figura 2 muestra la comparación de los valores del test flamenco (número de caídas) entre los grupos de edad y sexo. Los resultados mostraron un efecto de interacción estadísticamente significativo $(p<0,05)$. Posteriormente, las comparaciones por pares mostraron que las esquiadoras de 14 años tenían unos niveles estadísticamente significativos mejores que sus compañeras de 11-13 años $(p<0,05)$. En cuanto a la comparación por sexo, los resultados mostraron que los esquiadores de 14 años tenían unos valores estadísticamente mayores que las esquiadoras de su mismo grupo de edad $(p<0,05)$. 


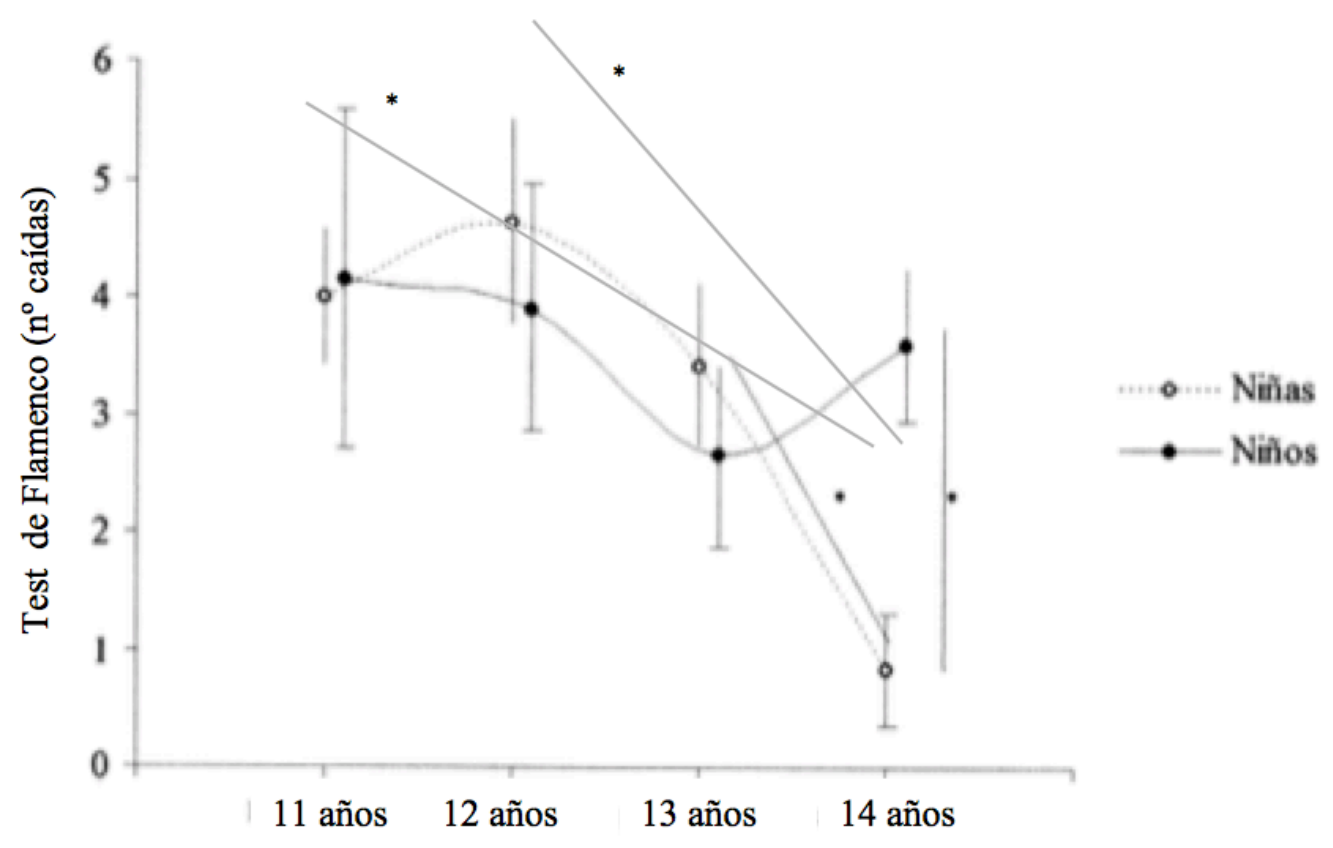

Figura 2. Comparación de los valores del test flamenco (número de caídas) entre los grupos de edad y sexo. Los valores representan la media y las barras de error el error estándar. Nivel de significación del análisis de la varianza de dos factores $(\mathrm{p}<0,05)$ seguido por las comparaciones por pares con la corrección de Bonferroni (sobre figura): $* p<0,05$.

La Figura 3 muestra la comparación de los valores del test del hexágono (s) entre los grupos de edad y sexo. Los resultados mostraron un efecto de interacción estadísticamente significativo $(p<0,05)$. Posteriormente, las comparaciones por pares mostraron que los esquiadores de 12 años tenían unos niveles estadísticamente significativos mejores que sus compañeros de 11 años $(p<0,05)$; las esquiadoras de 12,13 y 14 años tenían unos niveles estadísticamente significativos mejores que sus compañeras de 11 años $(p<0,05)$. 


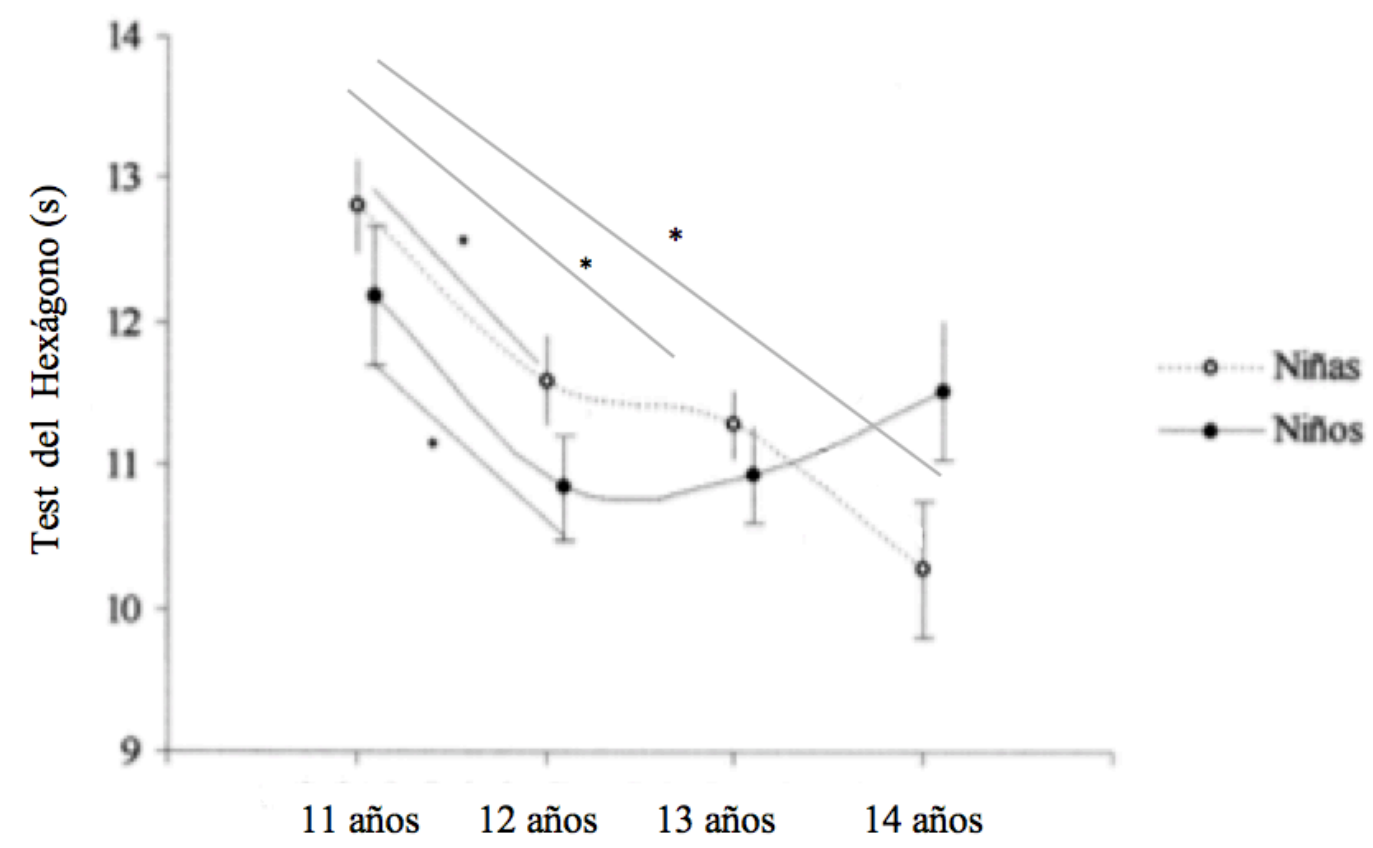

Figura 3. Comparación de los valores del test del hexágono (s) entre los grupos de edad y sexo. Los valores representan la media y las barras de error, el error estándar. Nivel de significación del análisis de la varianza de dos factores $(p<0,05)$ seguido por las comparaciones por pares con la corrección de Bonferroni (sobre figura): $* p<0,05$.

La Figura 4 muestra la comparación de los valores del test de Harre (s) entre los grupos de edad y sexo. Los resultados mostraron un efecto de interacción estadísticamente significativo $(p<0,05)$. Posteriormente, las comparaciones por pares mostraron que las esquiadoras de 14 años tenían unos niveles estadísticamente significativos mejores que sus compañeras de 11-13 años $(p<0,05)$. En cuanto a la comparación por sexo, los resultados mostraron que los esquiadores de 11 años tenían unos valores estadísticamente mayores que las esquiadoras de su mismo grupo de edad $(p<0,001)$. 


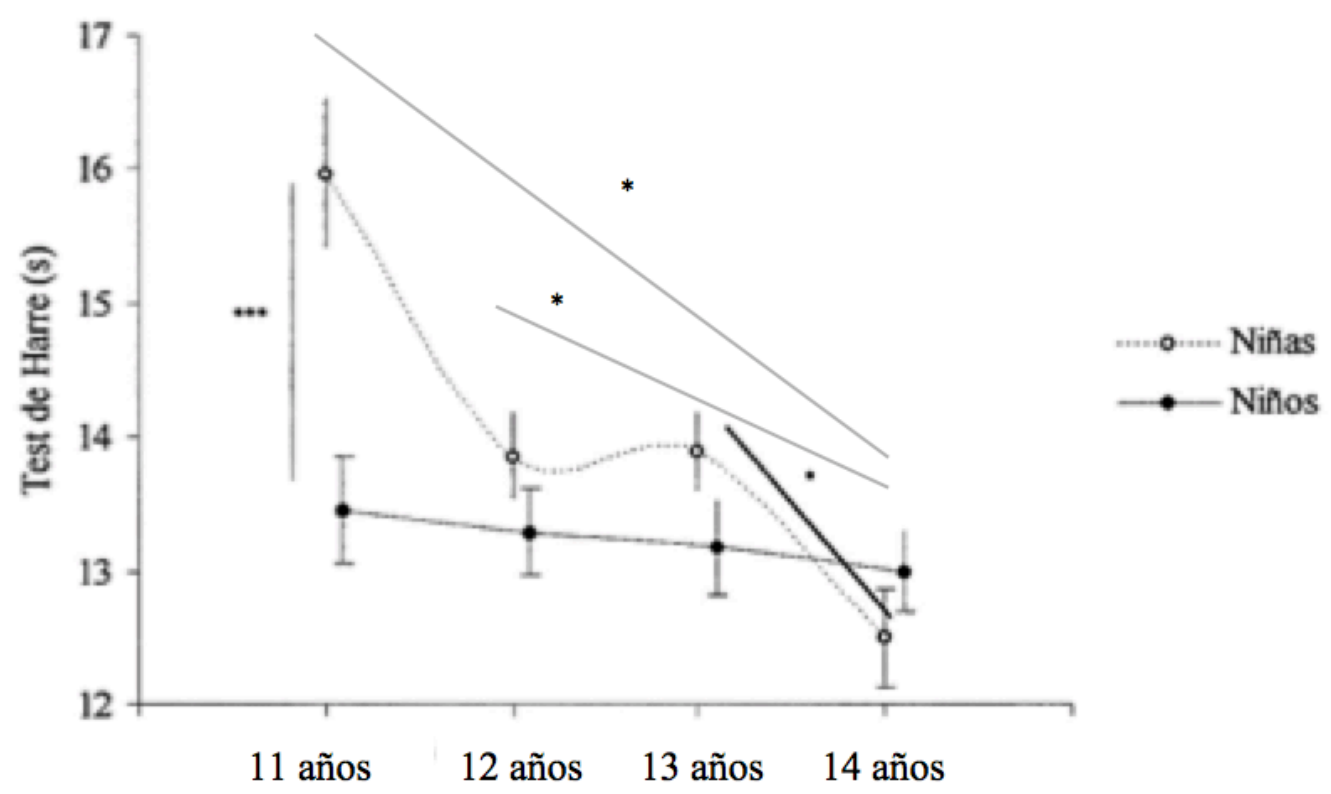

Figura 4. Comparación de los valores del test de Harre (s) entre los grupos de edad y sexo. Los valores representan la media y las barras de error el error estándar. Nivel de significación del análisis de la varianza de dos factores $(p<0,05)$ seguido por las comparaciones por pares con la corrección de Bonferroni (sobre figura): $* p<$ $0,05 \mathrm{y} * * * p<0,001$.

La Figura 5 muestra la comparación de los valores del test de $10 \times 5 \mathrm{~m}(\mathrm{~s})$ entre los grupos de edad y sexo. Los resultados mostraron un efecto de interacción estadísticamente significativo $(p<0,05)$. Posteriormente, las comparaciones por pares mostraron que los esquiadores de 11 años tenían unos valores estadísticamente significativos mejores que las esquiadoras de su mismo grupo de edad $(p<0,001)$. 


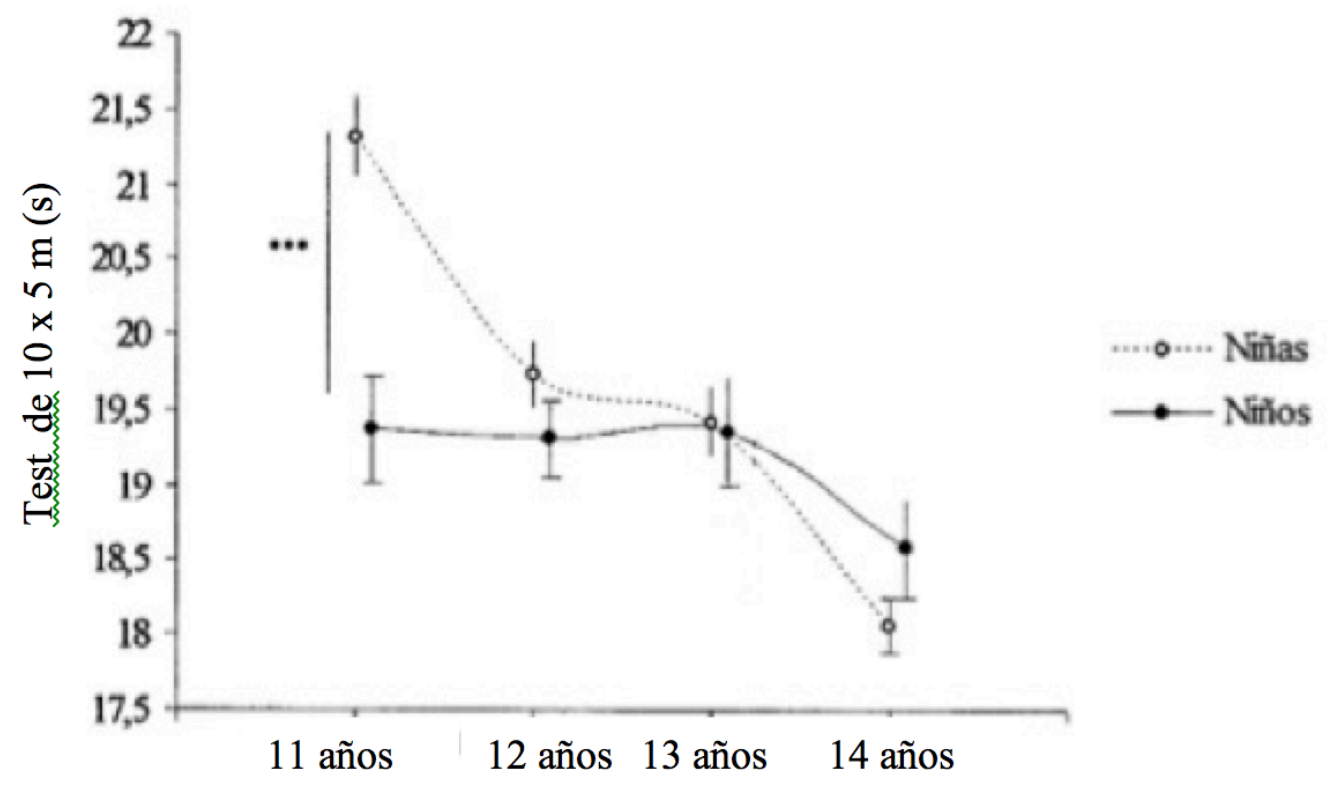

Figura 5. Comparación de los valores del test de 10 x 5 m (s) entre los grupos de edad y sexo. Los valores representan la media y las barras de error el error estándar. Nivel de significación del análisis de la varianza de dos factores $(p<0,05)$ seguido por las comparaciones por pares con la corrección de Bonferroni (sobre figura): $* * * p$ $<0,001$.

La Figura 6 muestra la comparación de los valores del test de salto de longitud (s) entre los grupos de edad y sexo. Los resultados mostraron un efecto de interacción estadísticamente significativo $(p<0,05)$. Posteriormente, las comparaciones por pares mostraron que a medida que aumentaban la edad las esquiadoras tenían unos niveles estadísticamente significativos mejores que el resto de sus compañeras de edades inferiores $(p<0,05)$, excepto para la comparación de 13 y 14 años que no fue estadísticamente significativa $(p>0,05)$; los esquiadores de 14 años tenían unos niveles estadísticamente significativos mejores que sus compañeros de 11 años $(p<0,001)$. En cuanto a la comparación por sexo, los resultados mostraron que los esquiadores de 11 años tenían unos valores estadísticamente mayores que las esquiadoras de su mismo grupo de edad $(p<0,01)$. 


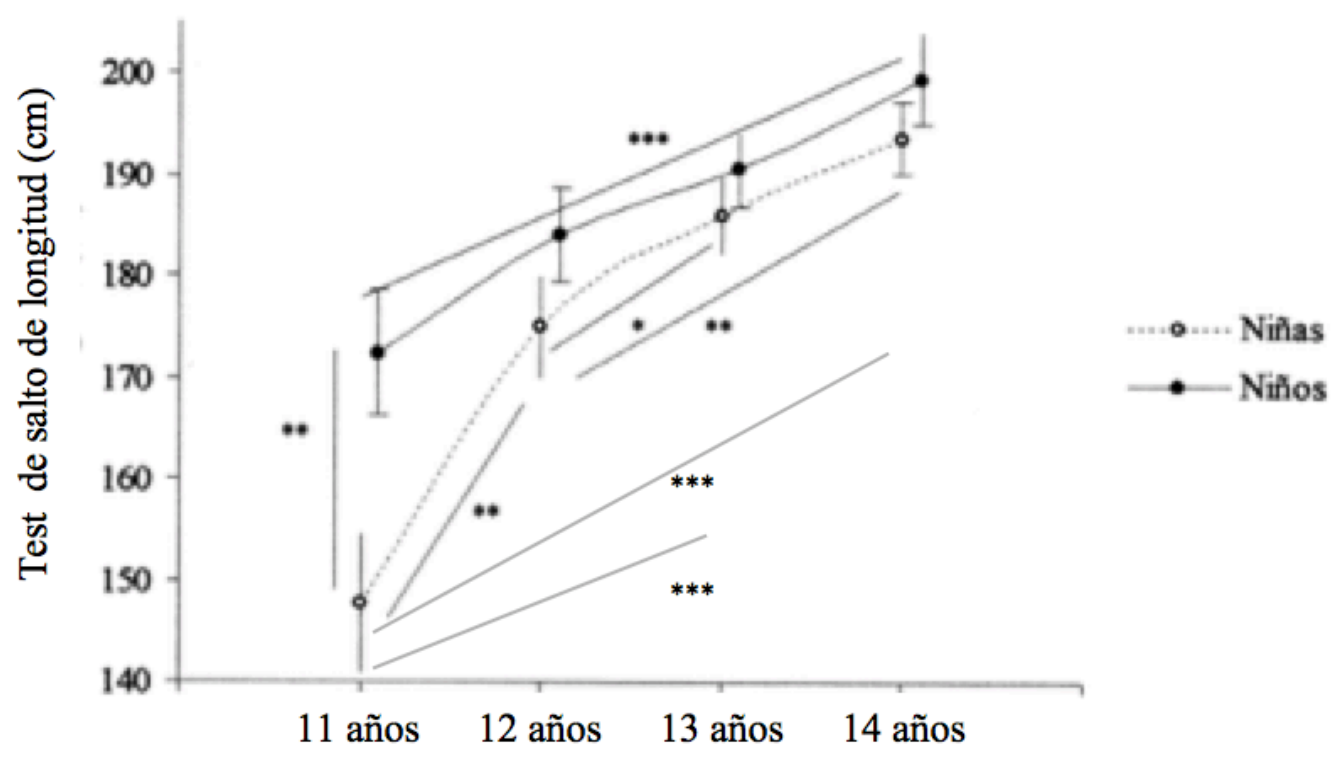

Figura 6. Comparación de los valores del test de salto de longitud (s) entre los grupos de edad y sexo. Los valores representan la media y las barras de error el error estándar. Nivel de significación del análisis de la varianza de dos factores $(p<0,05)$ seguido por las comparaciones por pares con la corrección de Bonferroni (sobre figura): $* p<0,05, * * p<0,01 \mathrm{y} * * * p<0,001$.

La Figura 7 muestra la comparación de los valores del test de Squat Jump (cm) entre los grupos de edad y sexo. Los resultados mostraron un efecto de interacción estadísticamente significativo $(p<0,05)$. Posteriormente, las comparaciones por pares mostraron que los esquiadores de 13 años tenían unos niveles estadísticamente significativos mayores que sus compañeros de 11 años $(p<0,001)$; las esquiadoras de 13 y 14 años tenían unos niveles estadísticamente significativos mayores que sus compañeras de 11-12 años $(p<0,05)$, así como que las mujeres de 11 años tenían unos valores estadísticamente mayores que sus compañeras de 12 años $(p<0,05)$. En cuanto a la comparación por sexo, los resultados mostraron que los esquiadores de 11-14 años tenían unos valores estadísticamente mayores que las esquiadoras de su mismo grupo de edad $(p<0,01)$. 


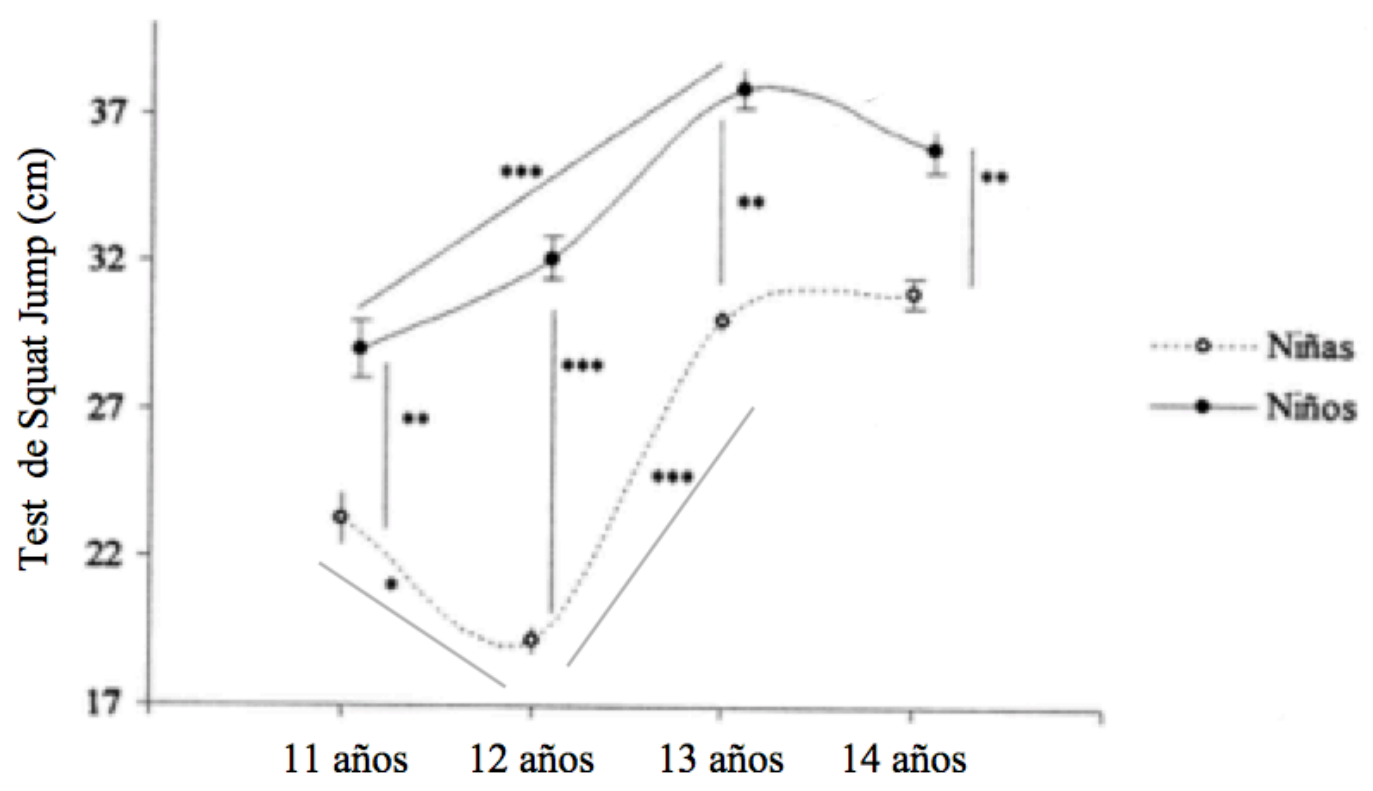

Figura 7. Comparación de los valores del test de Squat Jump (cm) entre los grupos de edad y sexo. Los valores representan la media y las barras de error el error estándar. Nivel de significación del análisis de la varianza de dos factores $(p<0,05)$ seguido por las comparaciones por pares con la corrección de Bonferroni (sobre figura): $* p<0,05, * * p<0,01 \mathrm{y} * * * p<0,001$.

La Figura 8 muestra la comparación de los valores del test de Counter Movement Jump $(\mathrm{cm})$ entre los grupos de edad y sexo. Los resultados mostraron un efecto de interacción estadísticamente significativo $(p<0,05)$. Posteriormente, las comparaciones por pares mostraron que los esquiadores de 13-14 años tenían unos niveles estadísticamente significativos mayores que sus compañeros de 11 años $(p<0,01)$; las esquiadoras de 13 y 14 años tenían unos niveles estadísticamente significativos mayores que sus compañeras de 11-12 años $(p<0,05)$, así como que las mujeres de 11 años tenían unos valores estadísticamente mayores que sus compañeras de 12 años $(p<0,05)$. En cuanto a la comparación por sexo, los resultados mostraron que los esquiadores de 11-14 años tenían unos valores estadísticamente mayores que las esquiadoras de su mismo grupo de edad $(p<0,01)$. 


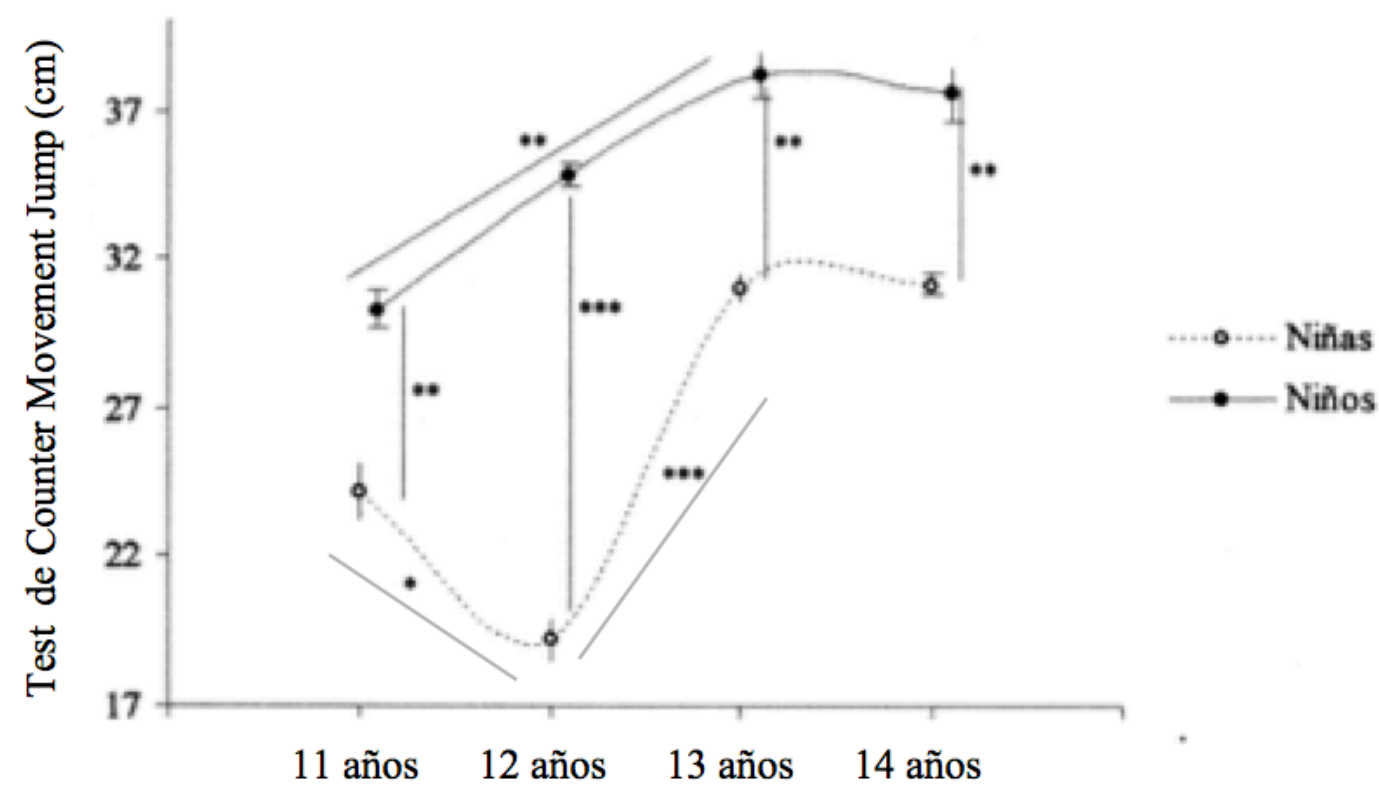

Figura 8. Comparación de los valores del test de Counter Movement Jump (cm) entre los grupos de edad y sexo. Los valores representan la media y las barras de error el error estándar. Nivel de significación del análisis de la varianza de dos factores $(p<0,05)$ seguido por las comparaciones por pares con la corrección de Bonferroni (sobre figura): * $p<0,05, * * p<0,01 \mathrm{y} * * * p<0,001$.

La Figura 9 muestra la comparación de los valores del test de Drop Jump (cm) entre los grupos de edad y sexo. Los resultados mostraron un efecto de interacción estadísticamente significativo $(p<0,05)$. Posteriormente, las comparaciones por pares mostraron que los esquiadores de 13-14 años tenían unos niveles estadísticamente significativos mayores que sus compañeros de 11-12 años $(p<0,01)$; las esquiadoras de 13 y 14 años tenían unos niveles estadísticamente significativos mayores que sus compañeras de 11-12 años $(p<0,05)$, así como que las mujeres de 11 años tenían unos valores estadísticamente mayores que sus compañeras de 12 años $(p<0,05)$. En cuanto a la comparación por sexo, los resultados mostraron que los esquiadores de 11-14 años tenían unos valores estadísticamente mayores que las esquiadoras de su mismo grupo de edad $(p<0,001)$. 


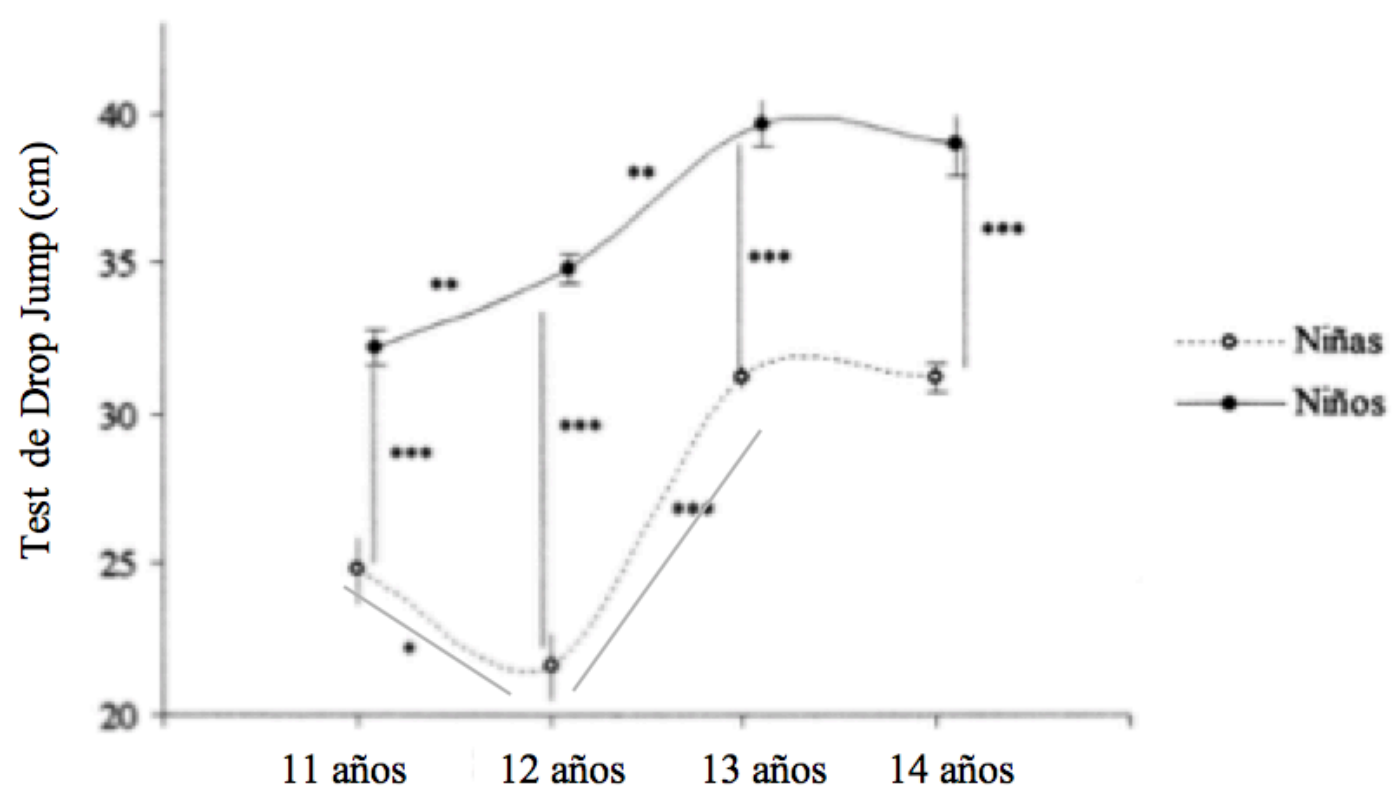

Figura 9. Comparación de los valores del test de Drop Jump (cm) entre los grupos de edad y sexo. Los valores representan la media y las barras de error el error estándar. Nivel de significación del análisis de la varianza de dos factores $(p<0,05)$ seguido por las comparaciones por pares con la corrección de Bonferroni (sobre figura): $* p<0,05, * * p<0,01 \mathrm{y} * * * p<0,001$.

La Figura 10 muestra la comparación de los valores del test de Counter Movement Jump $(\mathrm{CMj})$ en $15 \mathrm{~s}(\mathrm{~cm})$ entre los grupos de edad y sexo. Los resultados mostraron un efecto de interacción estadísticamente significativo $(p<0,05)$. Posteriormente, las comparaciones por pares mostraron que los esquiadores de 13 años tenían unos niveles estadísticamente significativos mayores que sus compañeros de 11 años $(p<0,001)$; las esquiadoras de 13-14 años tenían unos niveles estadísticamente significativos mayores que sus compañeras de 11-12 años $(p<0,05)$; las niñas de 11 años tenían unos valores estadísticamente mayores que sus compañeras de 12 años $(p<0,05)$ y las de 13 mayores que las de 14 años $(p<0,05)$. En cuanto a la comparación por sexo, los resultados mostraron que los esquiadores de 11-14 años tenían unos valores estadísticamente mayores que las esquiadoras de su mismo grupo de edad $(p<$ $0,01)$. 


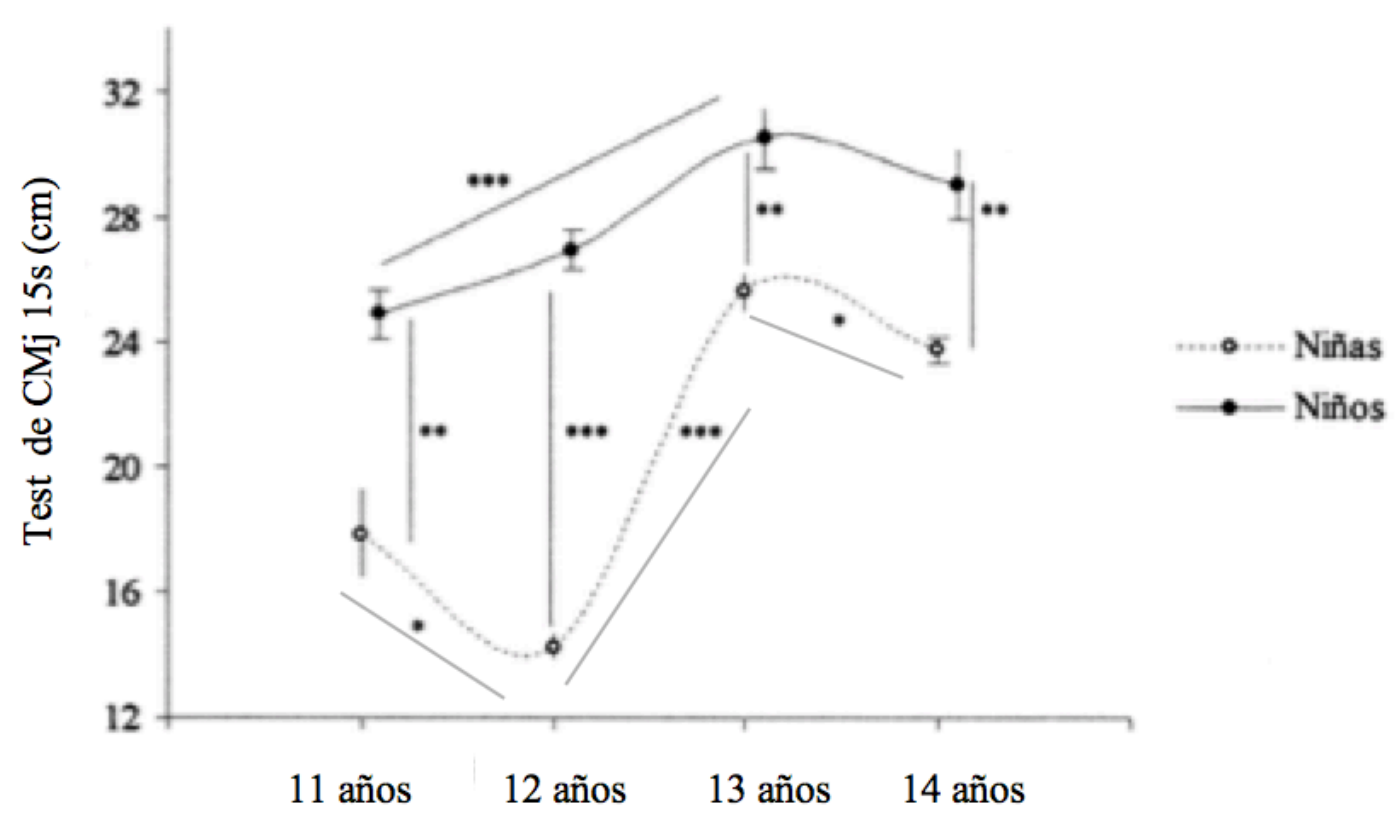

Figura 10. Comparación de los valores del test de Counter Movement Jump en $15 \mathrm{~s}(\mathrm{~cm})$ entre los grupos de edad y sexo. Los valores representan la media y las barras de error el error estándar. Nivel de significación del análisis de la varianza de dos factores $(p<0,05)$ seguido por las comparaciones por pares con la corrección de Bonferroni (sobre figura): $* p<0,05, * * p<0,01 \mathrm{y} * * * p<0,001$.

La Figura 11 muestra la comparación de los valores del test de Counter Movement Jump en $45 \mathrm{~s}(\mathrm{~cm})$ entre los grupos de edad y sexo. Los resultados mostraron un efecto de interacción estadísticamente significativo $(p<0,05)$. Posteriormente, las comparaciones por pares mostraron que las esquiadoras de 13-14 años tenían unos niveles estadísticamente significativos mayores que sus compañeras de 12 años $(p<0,001)$; en los esquiadores de diferentes edades no se encontraron diferencias estadísticamente significativas $(p>0,05)$. En cuanto a la comparación por sexo, los resultados mostraron que los esquiadores de 12 y 14 años tenían unos valores estadísticamente mayores que las esquiadoras de su mismo grupo de edad $(p<$ $0,05)$. 


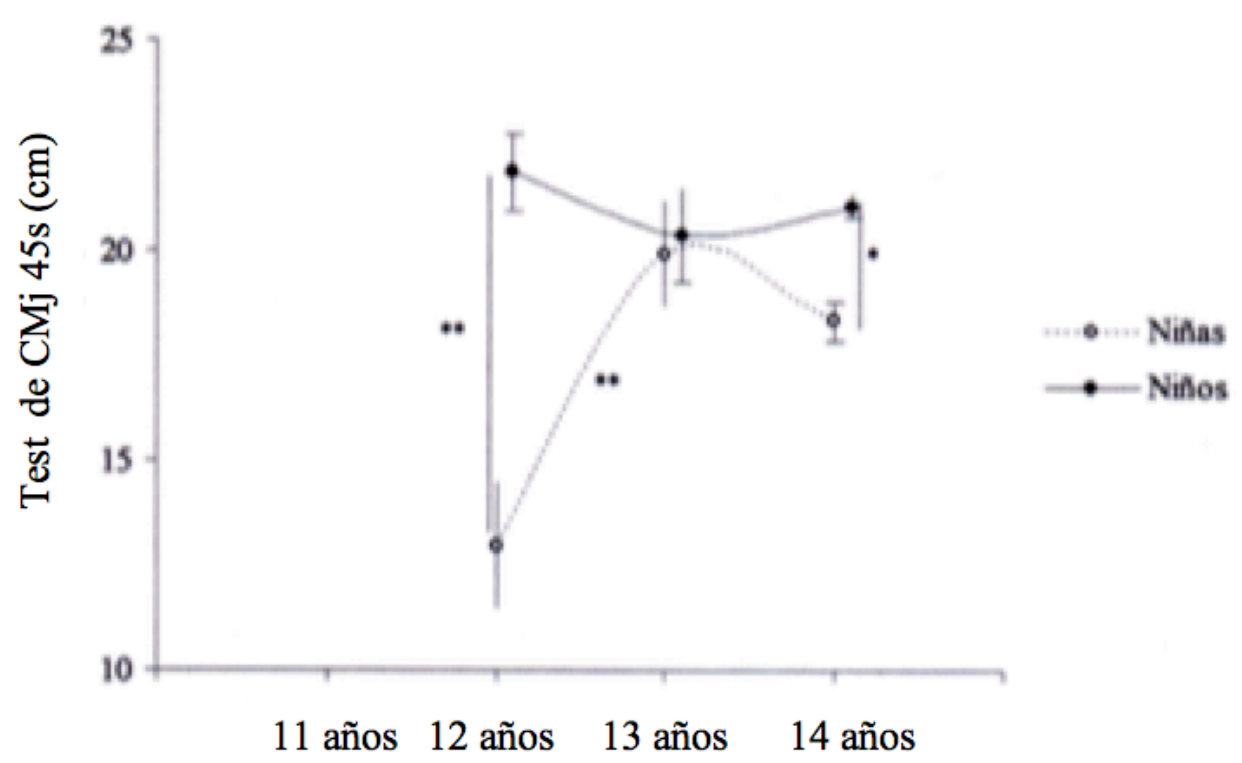

Figura 11. Comparación de los valores del test de Counter Movement Jump en $45 \mathrm{~s}$ (cm) entre los grupos de edad y sexo. Los valores representan la media y las barras de error el error estándar. Nivel de significación del análisis de la varianza de dos factores $(p<0,05)$ seguido por las comparaciones por pares con la corrección de Bonferroni (sobre figura): $* p<0,05 \mathrm{y} * * p<0,01$.

\section{DISCUSIÓN}

El primer objetivo del presente estudio fue mostrar los valores percentiles de los test motores de referencia en esquiadores españoles de categoría infantil (niños y niñas de 11-14 años). El segundo objetivo del este estudio fue comparar los niveles de rendimiento en los test motores entre los diferentes grupos de edad y sexo.

Los resultados obtenidos en el test de flexibilidad de tronco fueron similares a los encontrados en diversos estudios acerca de la batería Eurofit para la población general de niños (Prat, 1986; Council of Europe, 1993). En cambio, fueron muy superiores a los conseguidos por Merni y Carbonaro, (1983), con jóvenes deportistas italianos entre 11 y 14 años. La capacidad de flexibilidad mejoró con la edad tanto en niños como en niñas, encontrando valores moderadamente altos y grandes diferencias entre géneros. No obstante, se puede decir que las niñas presentaron mejores resultados que los niños, especialmente entre los 11 y 12 años.

Los resultados del test del flamenco no mostraron diferencia entre niños y niñas, salvo cuando éstas se estabilizaron tras la pubertad. Fue en este momento cuando presentaron mejores valores respecto a los niños. Si comparamos los valores medios de este grupo de jóvenes esquiadores con los obtenidos en población normal, encontramos valores superiores sobre todo en niñas esquiadoras de 14 años con respecto a las niñas en general, posiblemente debido a que ya a esta temprana edad, numerosas chicas se apartan de la actividad deportiva (Klika \& Malina, 1997; Raschner, Patterson, Platzer \& Lembert, 2008; Ruzic, Petracic \& Radenovic, 2011). 
Los resultados obtenidos del test del hexágono coinciden con los encontrados en estudios mencionados en el marco teórico en deportistas de estas edades (McGinnis, Piper \& Dillman, 1981; Reid, Kipp, Albert, White \& Johnson, 1997; Ruiz de Almirón et al., 2002) e incluso con deportistas de edades comprendidas entre los 12 a 19 años realizados con esquiadores americanos y noruegos (Dillman, 1990; Klika \& Malina, 1997; Heikkinen, 2003; NSF, 2014). Esto podría constatar el alto nivel de relación que tiene el test hexagonal con el rendimiento y su utilización como predictor del rendimiento en esquí alpino.

Los resultados obtenidos en el Test de Harre demostraron la importancia de la capacidad de coordinación dinámica general dentro del esquí alpino infantil habiendo presentado valores superiores a los de jóvenes deportistas de otras disciplinas, en ambos sexos. Este hecho coincide con los resultados obtenidos en estudios más recientes con el mismo test (Müller et al., 2015b; Steidl-Müller et al., 2017). Estos estudios confirman que esta capacidad motora no se ve afectada significativamente por la maduración en niños y la existencia de diferencias altamente significativas a los 11 años, dentro del intervalo comprendido entre los 12 y 14 años, en niñas. El motivo de estas diferencias entre niños y niñas podría estar relacionado con la diferente maduración biológica existente en estas edades y con el efecto de la edad relativa en los test motores y en el rendimiento en esquí alpino (Gorski et al., 2014; Müller et al., 2015a; SteidlMüller et al., 2017, 2019).

Los resultados obtenidos en el test de $10 \times 5 \mathrm{~m}$, fueron sensiblemente superiores a los de los niños en general, en ambos géneros, si los comparamos con los datos de estudios con población europea (Council of Europe, 1993; Ruiz et al, 2006), y similares a los obtenidos con esquiadores de élite nacional infantil española (Ruiz de Almirón et al, 2002). Esto podría indicar que el test de 10x5 m puede servir para predecir el rendimiento en esquí alpino desde la edad infantil en ambos géneros, coincidiendo con los tests similares de los modelos de detección descritos como el Zigzag run, Swiss cross y Aglility-T-test (Heikkinen, 2003; Gorski et al., 2013).

Los resultados del test de salto horizontal sugieren que el esquí alpino necesita altos valores de fuerza explosiva y capacidad motora general en la edad infantil. Estos mostraron similares resultados a los encontrados en niños no esquiadores (Council of Europe, 1993; Merni \& Carbonaro, 1983), sin embargo, demostraron resultados superiores en niñas esquiadoras. En nuestra opinión, puede ser debido a una mayor motivación hacia las actividades de desarrollo de la fuerza que presentan habitualmente los niños, a los requerimientos de fuerza propios del tren inferior obtenidos como consecuencia de la práctica del esquí alpino y a una menor implicación de las niñas en actividades que desarrollen la fuerza. Cuando se comparan los resultados del test de salto horizontal de niños esquiadores con los de niños no esquiadores, es cuando se hace más patente la importancia de la fuerza explosiva y total del tren inferior en el esquí alpino ya que los niveles medios son en ambos géneros casi un $10 \%$ superiores a los de los niños en general (Prat, 1986). Los valores encontrados fueron muy similares a los valores de otros estudios realizados con esquiadores (Gorski et al., 2014; NSF, 2014). Al igual que sucediera con test anteriores, estos resultados parecen demostrar la relación entre esta capacidad y el rendimiento en esquí alpino, así como la utilización del test del salto de longitud horizontal para evaluar esta capacidad al tratarse de un test sencillo, fácilmente reproducible en cualquier instalación y altamente correlacionado con el test de salto CMJ de la Batería de Test de Bosco (García López \& Herrero, 2004).

En cuanto a los resultados obtenidos en los test de SJ, CMJ y DJ, podemos decir que obtuvieron resultados similares entre ellos y con los encontrados en esquiadores italianos en estas edades y superiores (Bosco et al., 1994; Bosco, 1997, 2000). El índice de elasticidad, obtenido a partir de la diferencia entre CMJ y el SJ no supera el 9\% de la capacidad de fuerza 
relativa en esquiadores adultos (Bosco, 1994, 1997; Ruiz de Almirón et al., 2002; Raschner et al., 2008, 2013; Müller et al., 2015b; Steidl-Müller et al., 2017, 2019). Esto demostró que esta capacidad requiere niveles de fuerza explosiva y reactiva elevados durante los cambios de dirección en el esquí durante la bajada. Este hecho manifiesta la importancia que tiene el entrenamiento de estas manifestaciones de fuerza desde etapas iniciales y su relación con el rendimiento en este deporte. En el esquí alpino es muy importante saber utilizar la fuerza soportada al final de curva y aprovechar la reacción de los esquís con la nieve para realizar el cambio de cantos entre curvas sin que haya interrupción de movimiento, de forma continua en la bajada.

Por último, y en relación a los test de CMJ en 15 y $45 \mathrm{~s}$, los resultados mostraron diferencias muy significativas entre sexos en todo el intervalo de edad. Esto no significó que los resultados obtenidos por las esquiadoras infantiles fueran muy bajos respecto a la población de deportistas en edad infantil, sino que los esquiadores niños presentaron una resistencia a la velocidad muy superior a otros deportistas de su misma edad si los comparamos con los encontrados en algunos estudios en niños y deportes de equipo. En estos, solo encontraron una diferencia de seis $\mathrm{cm}$ de altura de salto, frente a la comparación entre niñas esquiadoras y practicantes de deportes colectivos cuyos valores fueron similares. (Bosco, Cotelli, Bonomi, Mognoni 6 Roi, 1994; Bosco, 1997). Teniendo en cuenta lo anteriormente citado, se puede considerar que la resistencia a la fuerza veloz es una capacidad determinante en el esquí alpino de competición (Bosco, 1997) desde el momento de la salida y en los primeros 15 segundos de duración de la bajada, coincidiendo con los resultados obtenidos en esquiadores junior (Álvarez-San Emeterio \& Badillo., 2010, 2011) y con el test de 20 segundos de saltos continuos sobre cajón de la batería Alpine Medal Test para esquiadores entre 13 y 19 años (Stielow, 2010).

La resistencia a la fuerza veloz en el test de CMJ de $45 \mathrm{~s}$, presentó una tendencia parecida. No obstante, los resultados de las niñas se igualaron en la edad de 13 años en contra de lo encontrado en el test anterior. Estas diferencias pudieron estar debidas a los diferentes momentos de maduración en niños y niñas de 12 y 13 años, y probablemente a la muestra reducida con la que se contó. Teniendo en cuenta que la resistencia a la velocidad medida con saltos continuos se considera como la capacidad más determinante en el esquí alpino (Bosco, 1997), encontramos en la literatura consultada diferentes formas de evaluarla con resultados similares a los nuestros como demuestran los estudios en edades juveniles y seniors de los principales programas de esquí alpino existentes. Entre ellos, destacamos las pruebas de saltos laterales sobre un cajón denominada Box Jump test, en donde los datos coinciden en gran medida con los nuestros, incluso utilizando distintos intervalos de duración como por ejemplo durante 40 segundos (Stielow, 2010), 60 segundos (BC Alpine, 2014) y 90 segundos (Gorski et al., 2014; Heikkinen, 2003; NSF, 2014; Stielow, 2010; Raschner et al., 2013; Turnbull et al., 2009). Si comparamos los resultados obtenidos por la muestra de esquiadores analizados en este test con otras actividades deportivas, podemos decir que mantienen igual magnitud que en las edades adultas, situando al esquí alpino entre las disciplinas que requieren en gran medida la resistencia a la velocidad para su rendimiento, siendo superado por jugadores de voleibol, marchadores y atletas de sprint, todos de nivel internacional (Bosco, 2000).

En cuanto a las limitaciones del estudio, cabe destacar la carencia de estudios de este tipo con esquiadores de edades comprendidas entre los 11 y 14 años con los que comparar nuestros resultados, ya que los existentes en el momento e incluso estudios posteriores fueron realizados con deportistas en edad juvenil y adulta e incluso con diferentes baterías de test. Por tanto, estudios futuros deberían repetir este análisis con el mismo grupo y comparar con los datos recogidos en 2000-2001 e incluso apostar por otros grupos de edad, quedando pendiente un análisis de regresión lineal para conocer qué test motores son los que mejor predicen el rendimiento deportivo, teniendo en cuenta los resultados de estos mismos participantes a lo 
largo de su vida deportiva o en una competición concreta como pudiera haber sido este campeonato. De esta forma se proporcionaría información muy útil para los entrenadores.

\section{CONCLUSIONES}

Existen numerosas diferencias en los valores de los test motores entre las categorías de edad y sexo en esquiadores de esquí alpino español de categoría infantil. La elección de las pruebas utilizadas para la predicción del rendimiento deportivo en edad infantil en este estudio puede ser útiles para evaluar las capacidades físicas, detectar talentos deportivos, y controlar el entrenamiento en el esquí alpino en la edad de 11-14 años.

\section{REFERENCIAS BIBLIOGRÁFICAS}

Álvarez-San Emeterio, C., \& González-Badillo, J. J. (2010). The physical and anthropometric profiles of adolescent alpine skiers and their relationship with sporting rank. Journal of Strength \& Conditioning Research, 24(4), 1007-1012. https//doi.org/10.1519/JSC.0b013e3181cbabb5

Bandalo, M., \& Lesnik, B. (2011). The connection between selected anthropometric and motor variables and the competitive success of young competitors in alpine skiing. Kinesiologia Slovenica, 17(3), 16-31.

Bosco, C. (1990). La fisiologia, pianificazione, valutazione e controllo della preparazione fisica nello sci alpino con il test Di Bosco. Milano: Sci Agnóstico (G. D’urbano Ed), Sperling \& Kupfer Editori.

Bosco, C. (1997). Evaluation and planning of conditioning training for alpine skiers. En E. Müller et al. (Eds.), Science and skiing I (pp 229-250). Hamburg: Verlag Dr. Kovac.

Bosco, C. (2000). La fuerza muscular: Aspectos metodológicos. Barcelona: Inde.

Bosco, C. F.; Cotelli, R.; Bonomi, P.; Mognoni, G. S., \& Roi G. H. (1994). Seasonal fluctuations of selected physiological characteristics of elite alpine skiers. European Journal of Applied Physiology, 69(1), 7174. https//doi.org/10.1007/BF00867930

Council of Europe. (1993). Handbook for the EUROFIT test of physical fitness. Strasbourg: Council of Europe, Committee for the Development of Sport.

Dillman, C.J., (1990). Test para el esquí alpino. En R. B. Arnot y C. L. Gaines (Eds.), Seleccione su deporte (pp. 225-246). Barcelona: Paidotribo.

García López, J., \& Herrero Alonso, J. A. (2004). Variables cinéticas de la batida relacionadas con el rendimiento del salto horizontal a pies juntos. Biomecánica: órgano de la Sociedad Ibérica de Biomecánica y Biomateriales. 2004, vol. 12, núm. 2.

Gómez-López, P. J.; Rupérez, O. H.; Ruiz de Almirón-Megías, P., \& Calderon Soto, C. (2012). The anthropometric and physiological profiles in Spanish downhill skiing competitors. In V. E. Müeller, S. Lindinger, \& T. Stöggl (Eds.), Science and skiing (pp. 192-201). Austria: Meyer \& Meyer Sport.

Gorski, T.; Rosser, T.; Hoppeler, H., \& Vogt, M. (2014). An anthropometric and physical profile of young Swiss alpine skiers between 2004 and 2011. International Journal of Sports Physiology and Performance, 9(1), 108-116. https//doi.org/10.1123/ijspp.2013-0223

Heikkinen, D. (2003). Physical testing characteristics and technical event performance of junior alpine ski racers (Unpublished doctoral dissertation). The University of Maine, Maine.

Klika, R. J., \& Malina, R. M. (1997). Predicting skiing performance in 14-18 year old competitive alpine skiers. En E. Müller (Ed.), Science and skiing I (pp. 272-284). Hamburg: Verlag Dr. Kovac.

McGinnis PM; Piper FC., \& Dillman CJ., (1981). Skills tests for discrimination of alpine skiing ability, University of Illinois Press, Urbana, Illinois.

Merni, F., \& Carbonaro, G. (1983). Test motori per la valutazione dei giovani dagli 11 ai 14 anni [Motor tests for the assessment of youths between 11 and 14 years]. Rome: CONI/SdS.

Müller, L.; Hildebrandt, C., \& Raschner, C. (2015a). The relative age effect and the influence on performance in youth alpine ski racing. Journal of Sports Science \& Medicine, 14(1), 16-22.

Müller, L.; Müller, E.; Kornexl, E., \& Raschner, C. (2015b). The relationship between physical motor skills, gender and relative age effects in young Austrian alpine ski racers. International Journal of Sports Science and Coaching, 10(1), 69-86. https//doi.org/10.1260/1747-9541.10.1.69

Neumayr, G.; Hoertnagl, H.; Pfister, R.; Koller, A.; Eibl, G., \& Raas, E. (2003). Physical and physiological factors associated with success in professional alpine skiing. International Journal of Sports Medicine, 24(8), 571-575. https//doi.org/10.1055/s-2003-43270

Norsk Skiforbund. (2014). Ironman Protokoller. Accedido 3 de octubre de 2015 desde http://www.skiforbundet.no/PageFiles/1238/IronmanProtokoller Ver5 140813.pdf 
Prat Subirana, J. A. (1986). El control deportivo escolar: Factores a controlar y formas de aplicación. En Jornadas de Medicina del Deporte (Tomo II) (pp. 641-699). Las Palmas de Gran Canaria: Consejería de Cultura y Deportes, Gobierno de Canarias.

Raschner, C.; Patterson, C.; Platzer, H. P., \& Lembert, S. (2008). Kraftleistungen österreichischer nachwuchskaderathleten im alpinen skirennsport -Normwerte einer längsschnittstudie über 10 jahre. En Bundesinstitut für Sportwissenschaft (Ed.), Krafttraining im Nachwuchsleistungssport - Theorie Trifft Praxis (pp. 67-86). Leipzig: Leipziger Verlagsanstalt GmbH.

Reid, R. C.; Kipp, R.W.; Albert, R. W.; White, A. T., \& Johnson, S. C. (1997). Validity of sport specific field tests for elite and developing alpine ski racers. In E. Muller, H. Schwameder, E. Kornexl, \& C. Raschner (Eds.), Science and skiing I (pp. 285-296). London, UK: E\&FN Spoon.

Ruiz, J. R.; Ortega, F. B.; Gutierrez, A.; Meusel, D.; Sjöström, M., \& Castillo, M. J. (2006). Health-related fitness assessment in childhood and adolescence: A European approach based on the AVENA, EYHS and HELENA studies. Journal of Public Health, 14(5), 269-277. https//doi.org/10.1007/s10389-0060059-z

Ruiz de Almirón, P.; Mesa-Mesa, J. L.; Gómez-López, P., y Gutiérrez Sainz, A. (2002). Detección de talentos y monitorización del entrenamiento en esquí alpino infantil. En M. Zabala, I. J. Chirosa, L. J. Chirosa, y J. Viciana (Eds.), Tecnología y metodología científica aplicada al control y evaluación del rendimiento deportivo (pp. 111-117). Granada: Servicio de Publicaciones de la Universidad de Granada.

Ruzic, L.; Petracic, T., \& Radenovic, O. (2011). The relationship between the field and the laboratory balance tests and skiing performance. Croatian Sports Medicine Journal, 1(26), 52-57.

Steidl-Müller, L.; Hildebrandt, C.; Müller, E.; Fink, C. \& Raschner, C., (2017). Long-Term Athletic Development in Youth Alpine Ski Racing: The Effect of Physical Fitness, Ski Racing Technique, Anthropometrics and Biological Maturity Status on Injuries. Frontiers in Physiology. 8. 656. 10.3389/fphys.2017.00656.

Steidl-Müller, L.; Hildebrandt, C.; Raschner, C. \& Müller, E., (2019) Challenges of talent development in alpine ski racing: a narrative review, Journal of Sports Sciences, 37:6, 601612, DOI: $10.1080 / 02640414.2018 .1513355$

Stielow, J. A. (2010). Establishment of physical assessment percentile ranks for junior alpine ski competitors (Unpublished doctoral dissertation). The University of Montana, Missoula.

Turnbull, J. R.; Kilding, A. E., \& Keogh, J. W. L. (2009). Physiology of alpine skiing. Scandinavian Journal of Medicine \& Science in Sports, 19(2), 146-155. https//doi.org/10.1111/j.1600-0838.2009.00901.x

United States Ski and Snowboard Association. (Producer). (2005). USSA Sport Performance Series. Alpine Training Environment [DVD]. USA: Big Rig Creative.

Warwitz, S. A. (1976). Das sportwissenschaftliche experiment: Planung, durchführung, auswertung, deutung. Schorndorf: Hofmann 